studies have indicated high diagnostic accuracy of ceVUS compared to VCUG in the detection of VUR, a limited ability for detecting IRR in children compared to VCUG has been suggested as a potential drawback of ceVUS. The aim of our study is to evaluate the prevalence of IRR diagnosed by ceVUS and its association with age, gender and grade of VUR.

We used LOGIQ S8 ultrasound machine equipped with dedicated software for contrast-enhanced studies and harmonic imaging (HI). Standard ultrasound of the urinary tract was followed by bladder catheterisation and instillation of physiological normal saline and the US contrast medium (SonoVue ${ }^{\circledR}$, Bracco). The diagnostic criterion for IRR is the appearance of contrast microbubbles outside the contours of the duct system or renal calyx and the entry of contrast into the renal parenchyma.

Since March 2006 until January 2020 we performed 8130 ceVUS examinations and found VUR in 2972 children (36,6\%). Until December 2012 we did not use contrast specific software and we found VUR in 1013 out of 2977 children $(34 \%)$ of whom IRR was found in 23 children $(2,3 \%)$. Since January 2013 we used contrast specific software and HI and found VUR in 1959 out of 5153 children (38\%) and IRR in 233 children (11,9\%). We detected IRR in 124 male $(53,2 \%)$ and 109 female patients $(46,8 \%)$ with age range from 15 days to 82 months. Most common indications for ceVUS were: febrile urinary tract infection (82\%), prenatally diagnosed hydronephrosis (14,6\%) and neurogenic bladder $(2,6 \%)$. Unilateral IRR was found in 180 patients $(77,3 \%)$ and bilateral in 53 patients $(22,7 \%)$. From all uretero-renal units with IRR (286), VUR was II grade in 8 children (2,8\%), III grade in $41(14,3 \%)$, IV grade in $174(60,8 \%)$ and $\mathrm{V}$ grade in 63 children $(22 \%)$.

Our results in the largest so far published series of patients with ceVUS show that using contrast specific software and harmonic imaging intrarenal reflux was found in about $12 \%$ of patients with VUR. IRR is found even in children with low grade reflux. Our results emphasize the importance of considering IRR in the future classification of VUR as well as in assessment of prognosis and therapy in each child with VUR.

\section{TRANSCUTANEOUS TIBIAL NERVE STIMULATION IN CHILDREN AND ADOLESCENTS WITH NEUROGENIC BLADDER AND BOWEL DYSFUNCTION}

\footnotetext{
${ }^{1,2}$ Andrea Cvitković Roić*, ${ }^{1}$ Alemka Jaklin Kekez, ${ }^{1}$ Marinela Škunca, 'Žaklina Smiljanec, ${ }^{1}$ Iva Palčić, ${ }^{1}$ Vesna Kumanović. 'Helena Clinic for Pediatric Medicine, Kneza Branimira 71, Zagreb, Croatia; ${ }^{2}$ Faculty of Medicine, Josip Juraj Strossmayer University of Osijek, Josipa Huttlera 4, 31000 Osijek, Croatia
}

\subsection{6/archdischild-2021-europaediatrics.382}

Transcutaneous tibial nerve stimulation (TTNS) is a non-invasive neuromodulatory method which is used mostly in children and adults with overactive bladder, nonobstructive urinary retention, functional obstipation, fecal incontinence and in chronic pelvic pain syndrome. Experience in treatment of children and adolescents with neurogenic dysfunction is very limited. Aim of this study is to evaluate the success of 3 months home TTNS therapy in patients with neurogenic bladder/bowel dysfunction.
In our institution in 2015 we started the program of noninvasive neuromodulation in treatment of children with different forms of lower urinary tract and bowel dysfunctions. In 75 of children with neurogenic bladder/bowel some form of neuromodulation was performed. In 36 of children some other treatments that could influence bladder/bowel function were performed together with TTNS so they were excluded from this study. We followed 39 patients in whom home treatment with TTNS was performed for 30 minutes every day during 12 weeks. Self-adhesive surface electrode was placed $4-5 \mathrm{~cm}$ cranial from medial malleolus. We evaluate treatment success by bladder/bowel symptom questionnaire before and after 12 weeks of therapy.

Out of 39 patients 23 had myelomeningocele, 9 had operation of spinal cord or pelvic tumor, 5 had tethered cord syndrome, 1 patient had spinal cord injury and 1 had transverse myelitis. There were 19 male and 20 female patients with age range from 4 to 21 years, average age 13,03+/- 5,04 years. Eight patients (20\%) did not feel any urinary/bowel symptom improvement. Twenty (51,3\%) patients had better sensation of bowel fullness, $2(30,8 \%)$ achieved stool continence, $12(30,8)$ had better sensation of bladder fullness, $6(15,4 \%)$ achieved urinary continence and $4(10,2 \%)$ reported voiding with less straining. None of our patients had side effects of TTNS.

Neuromodulation is a new method in patients with bladder/bowel dysfunction. TTNS is least invasive and might be valuable option for patients with neurogenic diseases. Although the method is not as effective as in children with non-neurogenic dysfunctions, most of our patients (80\%) had some symptom improvement, mostly related to bowel function. Our data in small group of patients suggest that transcutaneous electrical nerve stimulation might be effective and safe for treating patients with neurogenic disorders with positive effects especially on bowel function. Further larger welldesigned randomised controlled trials are needed to make definitive conclusions and evaluate long-term effects.

\section{ULTRASOUND ASSESSMENT OF KIDNEY LENGTH WITH DUPLEX COLLECTING SYSTEM IN INFANTS}

Kristina Drnasin*. Former "Specialist paediatric surgery prim.dr.sc. Kristina Drnasin, MD spec. of Paediatrics, Solin ', Croatia

\subsection{6/archdischild-2021-europaediatrics.383}

Objective To determine the ratio of the sizes of kidneys with duplex collecting system (DCS) and healthy kidneys with one collecting system as well as kidneys with single collecting system located on the contralateral side of the kidney with DCS. Methods The study was conducted on healthy infants, born on time, who were from 30 to 210 days of age. Ultrasonic measurements of kidney lengths were taken on the group of 33 infants with DCS (14 males, 19 females) with 66 kidneys (DCS group) and 948 infants with 1896 healthy kidneys with single collecting system who were the control group (CG). Five of the 33 infants with DCS had bilateral DCS, which is total of 38 kidneys with DCS. The other 28 infants from the DCS group had one kidney with single collecting system, so this group is called the CDCS group.

Results We determined the normal values of kidney lengths of infants CG in relation to age, gender and body side and presented on four different nomograms of kidney length. 